\title{
Unification in intermediate logics
}

\author{
Rosalie Iemhoff* and Paul Rozière
}

October 21, 2013

\begin{abstract}
This paper contains a proof theoretic treatment of some aspects of unification in intermediate logics. It is shown that many existing results can be extended to fragments that at least contain implication and conjunction. For such fragments the connection between valuations and most general unifiers is clarified, and it is shown how from the closure of a formula under the Visser rules a proof of the formula under a projective unifier can be obtained. This implies that in the logics considered, for the $n$-unification type to be finitary it suffices that the $m$-th Visser rule is admissible for a sufficiently large $m$. At the end of the paper it is shown how these results imply several well-known results from the literature.
\end{abstract}

Keywords: unification, admissible rules, intermediate logics, fragments.

$M S C$ : 03B20, 03B55, 03B70, 03F03.

\section{Introduction}

Unification theory is the study of substitutions that identify two terms against a background theory of equality. In this paper the background theories are fragments of propositional intermediate logics that contain at least implication and conjunction. In this setting unification becomes the study of substitutions under which a formula becomes provable in a logic, in which case the substitutions are called the unifiers of the formula. This paper presents a proof-theoretic treatment of unification in these logics. It originates from the unpublished $\mathrm{PhD}$ thesis [30] by the second author, while the first author obtained new proofs of the theorems in [30] and thereby strengthened and simplified the results. In this introduction we will explain what these results are and discuss related work in the area. We start by explaining what unification types and admissible rules are.

In intermediate logics, any consistent formula is classically valid under some valuation (assigning 0 or 1 to the atoms). This valuation corresponds to a unifier of the formula, by taking $T$ for 1 and $\perp$ for 0 . Thus the existence of

\footnotetext{
* Support by the Netherlands Organisation for Scientific Research under grant 639.032.918 is gratefully acknowledged
} 
a unifier is equivalent to the consistency of the formula. Finding a maximal unifier for a formula is less trivial. A substitution is a maximal unifier $(m u)$ of a formula if among the unifiers of the formula it is maximal in the following ordering:

$$
\tau \leqslant \sigma \equiv_{\text {def }} \exists \pi\left(\tau={ }_{\mathrm{L}} \pi \sigma\right),
$$

and it is a most general unifier $(m g u)$ if it is also unique modulo $=_{\mathrm{L}}$. Here $=_{\mathrm{L}}$ is the equivalence relation on substitutions associated with the logic: $\sigma={ }_{\mathrm{L}} \tau$ if and only if $\sigma(p) \leftrightarrow \tau(p)$ is derivable for all atoms $p$. If $\tau \leqslant \sigma$ we say that $\tau$ is less general than $\sigma$. Mgus generate all unifiers of a formula, which is the reason that they play an important role in unification theory. The mgus that are important in the setting of logics are projective unifiers. $\sigma$ is a projective unifier $(p u)$ of a formula $A$ if it is a unifier of $A$ and $A$ implies that $\sigma$ is the identity:

$$
\forall p: A \vdash \sigma(p) \leftrightarrow p
$$

In this case $A$ is called projective. Projective unifiers were first introduced by Wroǹski under the name transparent unifiers [34], and they form one of the key notions in unification theory in logic.

In classical propositional logic every consistent formula has a mgu, but in nonclassical logics this no longer is the case. In intuitionistic logic $p \vee \neg p$ is an example of such a formula: the two valuations that classically satisfy this formula correspond to substitutions $\sigma_{1}(p)=\top$ and $\sigma_{2}(p)=\perp$, that cannot both be less general than another unifier. For if $\tau$ is a unifier of $p \vee \neg p$, the logic derives either $\tau p$ or $\neg \tau p$ by the disjunction property. Therefore either $\sigma_{1} \leqslant \tau$ or $\sigma_{2} \leqslant \tau$ holds, but not both. Hence $p \vee \neg p$ has no mgu.

The phenomenon that certain unification problems do not have mgus gave rise to the definition of unification types, which classify theories according to the existence or non-existence of mgus and mus. Although this definition applies to all theories, here we restrict ourselves to logics. A complete set of unifiers for a formula is a set of unifiers such that every unifier of the formula is less general than a unifier in the set.

A logic has n-unification type unitary if every unifiable formula of size (number of symbols) at most $n$ has a mgu and finitary if every unifiable formula of size at most $n$ has a finite complete set of mus. It has unification type unitary if its $n$-unification type is unitary for all $n$, and similarly for the finitary unification type. The other two types, infinitary (every unifiable formula has a (in)finite complete set of mus) and nullary (some unifiable formula does not have mus) will not be discussed.

As it turns out, many modal and intermediate logics have finitary unification, and this paper provides a proof-theoretic proof of this fact for intermediate logics. We use a notion that is closely related to unification types, that of admissible rules. A multi-conclusion rule $\Gamma / \Delta$ for finite sets of formulas $\Gamma$ and $\Delta$ is admissible, written $\Gamma \sim_{\mathrm{L}} \Delta$, if and only if

$$
\forall \sigma\left(\forall A \in \Gamma \vdash_{\mathrm{L}} \sigma A \Rightarrow \exists A \in \Delta \vdash_{\mathrm{L}} \sigma A\right)
$$


Thus a rule is admissible if it can be added to the logic without leading to new theorems, and the set of admissible rules form the largest class of inferences allowed to obtain the theorems of a logic and nothing more. It may be useful to know the admissible rules of a logic for various reasons. They may shorten proofs, as is the case for the cut rule in many Gentzen calculi. Or they may express properties of the logic that are intuitive yet invisible in standard axiomatizations, such as the disjunction property in the case of intuitionistic logic. The description of the admissible rules of a logic is usually given via a basis, which basically means a set of rules that are admissible and derive all other admissible rules of the logic.

In recent years, these two notions, unification and admissible rules, have been shown to be intimately connected, starting from the pioneering work of Ghilardi. In [10] Ghilardi proves that IPC has finitary unification by showing that every formula has a finite projective approximation $\Pi_{A}$, which is a finite set of projective formulas such that $\bigvee \Pi_{A} \vdash A \sim_{\mathrm{IPC}} \Pi_{A}$. Note that this indeed implies that $A$ has a finite complete set of maximal unifiers, namely the projective unifiers of the formulas in its projective approximation. The proofs in this paper that certain intermediate logics and fragments thereof have finitary or unitary unification follow the same pattern. By showing the stronger $\bigvee \Pi_{A} \vdash_{\mathrm{L}} A \vdash_{\mathrm{L}}^{\mathcal{R}} \Pi_{A}$, for some particular set of admissible rules $\mathcal{R}$, we simultaneously prove that $\mathcal{R}$ is a basis for the admissible rules of $L$. Here $\vdash_{L}^{\mathcal{R}}$ denotes derivability in $L$ extended by the rules $\mathcal{R}$. In this way results on unification go hand in hand with results on admissible rules.

What is new in this paper is the way in which it is proved that the formulas in the projective approximation are projective. This is related to the way the projective formulas are obtained. First we show, in the style of [19], that for every formula $A$ there is a set of formulas $\Pi_{A}$, the irreducible projective approximation of $A$, such that $\bigvee \Pi_{A} \vdash_{\mathrm{L}} A \vdash_{\mathrm{L}}^{\bar{\nabla}} \Pi_{A}$ and every formula in $\Pi_{A}$ is closed under the Visser rules $\bar{V}$. The Visser rules will be introduced in Section 6 and closure under a rule means that if all formulas in the antecedent are derived, then so is one of the conclusions. Then we show that the formulas in $\Pi_{A}$ are projective by showing that they are strongly satisfiable (to be defined below) and that, Theorem 1, strong satisfiability implies projectivity. The combination of these results imply that if the Visser rules are admissible, they form a basis and the logic has finitary or unitary unification.

The use of strong satisfiability also clarifies the connection between classical valuations and projective unifiers. Ghilardi also uses valuations in [12], but the link with projective unifiers is, we think, less direct than in the results above. In IPC the condition of strong satisfiability is also necessary for projectivity, and therefore it can be viewed as an analogue of Ghilardi's semantical characterization of projective formulas.

Most theorems in this paper apply to any fragment of any intermediate logic that contains implication and conjunction. In this way some of the known results on unification types and admissibility in intermediate logics are generalized. But rather than this slight generalization, which is not very surprising or particularly 
useful, we think the merit of this approach is its wide applicability, which stems from the fact that the proofs are purely syntactic and self-contained. It also provides short proofs of various other results on unification and admissible rules. We close the introduction with a discussion of related work on unification.

We saw that classical logic has unitary unification type and any intermediate logic with the disjunction property does not. Ghilardi [10] proved that intuitionistic propositional logic has finitary unfication, and he and Wroński [34] respectively proved that De Morgan Logic and Gödel-Dummett logic have unitary unification. Intermediate logics of nullary type are also known to exist [13]. Whether there is an intermediate logic with infinitary unification type is not known. Dzik located logics with unitary unification in the lattice of intermediate logics by showing that all extensions of De Morgan logic have nullary or unitary unification, and that for the latter the converse holds too [6]. There exist modal logics for which the unification problem is undecidable [33], but whether there exists an intermediate logic with this property is unknown. Rybakov considered, for intuitionistic and De Morgan logic, unification with parameters, which means that certain variables may only be substituted by themselves and showed that for this notion the unification type of these logics is finitary [32].

For fragments the situation is as follows. Prucnal [27] proved that the implication fragment of IPC has unitary unification, and the same holds for the implication-conjunction and the implication-conjunction-negation fragment of any intermediate logic [23]. The implication-negation fragment, however, has finitary instead of unitary unification, as was shown by Cintula and Metcalfe [4], who in the same paper also give a basis for the admissible rules of that fragment.

The paper is built-up as follows. Section 2 contains preliminaries and Section 3 explains the idea behind the proof of the main Theorem 2. Section 4 contains the technicalities needed to prove Theorem 1, which is done in Section 5. In Section 6 the relation between the Visser rules and strong satisfiability is established, which in Section 7 is used to obtain results about projective approximations, which imply results on unification in Section 8.

We would like to thank George Metcalfe, Jeroen Goudsmit and Emil Jeřábek for the fruitful discussions on unification and admissible rules that we have had. We also thank an anonymous referee for numerous helpful comments.

\section{Preliminaries}

Let $\mathcal{L}$ be a language for propositional logic with propositional variables, or atoms, $\mathcal{P}=\left\{p_{1}, p_{2}, \ldots\right\}$. $p, q, r, s$ denote arbitrary elements of $\mathcal{P} \cup\{\perp\}$, and in case that $\perp$ is not part of the language, of $\mathcal{P}$. In this paper $L$ can be any fragment of any propositional intermediate logic that contains the $\{\wedge, \rightarrow, \top\}$ fragment of intuitionistic logic IPC. $\top$ is not strictly necessary, but it will be convenient to have a separate symbol for truth available. When we speak of a fragment of a logic we include the possibility that the fragment is the full logic. 
Therefore all intermediate logics are among the logics we consider. $\vdash_{\mathrm{L}}$ stands for derivability in logic L. We use $\vdash$ when it is not important which particular logic we are considering.

We use $\Gamma, \Pi, \Delta, \Sigma$ to denote finite sets of formulas. Sequents are expressions $\Gamma \Rightarrow \Delta$. In the case that $\perp$ and negation do not belong to the language, we require that $\Delta$ is not empty, and in the case that disjunction does not belong to the language, we require that $|\Delta| \leq 1$. $S, R$ range over sequents. Given a subset $\mathcal{L}^{\prime}$ of $\mathcal{L}$, a formula, sequent or set of sequents is in $\mathcal{L}^{\prime}$ if all symbols in it belong to $\mathcal{L}^{\prime}$.

Atomic implications are implications of the form $p \rightarrow q$, where $p \neq \perp$. Note that $\neg p$ is an atomic implication. $\mathcal{L}_{n}$ is $\mathcal{L}$ minus the atoms $p_{i}$ for $i>n$. Given a set of sequents $\mathcal{G}, \mathcal{L}_{\mathcal{G}}$ denotes $\mathcal{L}$ minus the atoms that do not occur in $\mathcal{G}$ and $n_{\mathcal{G}}$ is the number of atoms in $\mathcal{G}$. For a sequent $S, i(S)$ denotes the number $\left(n_{\{S\}}+2 m\right)^{2}$, where $m$ is the number of implications in $S$, and similarly for formulas.

A sequent $(\Gamma \Rightarrow \Delta)$ is irreducible if $\Delta$ is empty or consists of atoms, and $\Gamma$ is empty or consists of atoms and atomic implications. $\mathcal{S}_{\mathcal{G}}$ is the set of irreducible sequents in $\mathcal{L}_{\mathcal{G}} \cdot \operatorname{var}(A)$ is the set of atoms that occur in $A$ and similarly for sequents and sets of sequents. In general, we will use $\mathcal{S}$ and $\mathcal{T}$ for arbitrary finite sets of sequents and $\mathcal{G}$ and $\mathcal{H}$ for finite sets of irreducible sequents.

We need the following notation, where $v$ stands for variable, $i$ for implication, $a$ for antecedent, and $c$ for conclusion:

$$
\begin{array}{cc}
(\Gamma \Rightarrow \Delta)^{a} \equiv_{d e f} \Gamma & (\Gamma \Rightarrow \Delta)^{c} \equiv_{d e f} \Delta \\
\Gamma^{v} \equiv_{d e f}\{p \mid p \in \Gamma\} & \Gamma^{i} \equiv_{d e f} \Gamma \backslash \Gamma^{v} \\
\Gamma^{a} \equiv_{d e f}\{p \mid \exists q(p \rightarrow q) \in \Gamma\} & \Gamma^{c} \equiv_{d e f}\{q \mid \exists p(p \rightarrow q) \in \Gamma\} \\
\Pi_{\mathcal{S}} \equiv_{d e f} \bigcup\left\{S^{a i} \mid S \in \mathcal{S}\right\} \Sigma_{\mathcal{S}} \equiv_{d e f}\left\{p \mid \mathcal{G} \vdash \Pi_{\mathcal{S}} \Rightarrow p\right\} \Gamma_{\Delta}^{i} \equiv_{d e f}\{(p \rightarrow q) \in \Gamma \mid p \in \Delta\} .
\end{array}
$$

For $k \in\{a, c\}$ and $l \in\{a, c, i, v\}, S^{k l}$ abbreviates $\left(S^{k}\right)^{l}$.

Sequents are interpreted in the usual way: $I(S)=\left(\bigwedge S^{a} \rightarrow \bigvee S^{c}\right)$, where an empty conjunction is $T$ and an empty disjunction $\perp$. For notational convenience we sometimes write $S$ for $I(S)$, for example in $\vdash S$, which thus should be read as $\vdash I(S)$. Sets of sequents are interpreted as conjunctions:

$$
I(\mathcal{G}) \equiv_{d e f} \bigwedge_{S \in \mathcal{G}} I(S)
$$

interpreting empty disjunctions by $\perp$ and empty conjunctions by $\top$. For sets of irreducible sequents $\mathcal{G}$ we sometimes denote $I(\mathcal{G})$ by the noncalligraphic version of the name of the set. For example, $G \equiv_{\text {def }} I(\mathcal{G})$ and $G_{i} \equiv_{\text {def }} I\left(\mathcal{G}_{i}\right)$. When we speak of the (in)consistency of $\mathcal{G}$, we mean the (in)consistency of $G$.

We use $\sigma$ and $\tau$ to denote substitutions, which are maps from propositional formulas to propositional formulas that commute with the connectives. $\iota$ is the identity substitution. As usual, $\tau \Gamma=\{\tau A \mid A \in \Gamma\}$ and $\tau S=\left(\tau S^{a} \Rightarrow\right.$ 
$\tau S^{c}$ ). Throughout the paper substitutions are assumed to have finite domains, denoted by $\operatorname{dom}(\cdot)$. We use the following notation for substitutions with the same domain:

$$
\sigma \leftrightarrow \tau \equiv_{\text {def }} \bigwedge_{p \in \operatorname{dom}(\sigma)} \sigma(p) \leftrightarrow \tau(p) .
$$

Observe that

$$
\vdash \sigma \leftrightarrow \tau \text { implies } \vdash \sigma A \leftrightarrow \tau A .
$$

Given two finite sets of sequents $\mathcal{T}$ and $\mathcal{T}^{\prime}, \mathcal{S}$ is closed under the multi-conclusion rule $\mathcal{T} / \mathcal{T}^{\prime}$ if whenever $I(\mathcal{S})$ derives $I(\mathcal{T})$ it derives $I(S)$ for at least one sequent $S$ in $\mathcal{T}^{\prime}$. The rule is admissible if for all substitutions $\sigma, \mathcal{S}$ is closed under the rule $\sigma \mathcal{T} / \sigma \mathcal{T}^{\prime}$. In the single-conclusion case, which means the case that $\left|\mathcal{T}^{\prime}\right| \leq 1$, $\mathcal{T} / \mathcal{T}^{\prime}$ is derivable if $I(\mathcal{S})$ derives $I(\mathcal{T}) \rightarrow I\left(\mathcal{T}^{\prime}\right)$. The same notions apply to logics by considering a logic $L$ as the set of its theorems. In this case we write $\mathcal{T} \sim_{L} \mathcal{T}^{\prime}$ if the rule is admissible, and $\mathcal{T} \vdash_{L} \mathcal{T}^{\prime}$ if the rule is derivable.

\section{Proof idea}

The main technical part of the paper is the proof of Theorem 2, which is divided in two parts, the proof of Theorem 1 and the proof of Lemma 11. In this section we briefly explain these proofs in general, the next three sections provide the technical details.

The proofs make use of a connection between valuations and substitutions that go back to Prucnal $[27,29]$ and are a crucial in the work of Ghilardi [10] and Rozière [30].

Given a set of atoms $I$ and a formula $A$ we define the valuation $v_{I}$ and substitution $\sigma_{I}$ as follows:

$$
v_{I}(p) \equiv_{d e f}\left\{\begin{array} { l l } 
{ 1 } & { \text { if } p \in I } \\
{ 0 } & { \text { if } p \notin I }
\end{array} \quad \sigma _ { I } ^ { A } ( p ) \equiv _ { \text { def } } \left\{\begin{array}{ll}
A \rightarrow p & \text { if } p \in I \\
A \wedge p & \text { if } p \notin I .
\end{array}\right.\right.
$$

It is not difficult to see that in classical logic $v_{I}(A)=1$ implies $\vdash_{\mathrm{CPC}} \sigma_{I} A$. This no longer holds in IPC, the formula $(p \rightarrow q \vee r)$ and $I=\{q\}$ being a counter example. There is, however, a weaker form of the statement that does hold. For a certain composition $\sigma_{A}$ of substitutions of the form $\sigma_{I}^{A}$ and a certain notion of satisfiablity called strong satisfiability, it is shown in Theorem 1 that if $A$ is strongly satisfiable, then $\sigma_{A}$ is a unifier for $A$. As $\sigma_{A}$ is a composition of $\sigma_{I}^{A}$ 's, it is a projective unifier of $A$. Thus showing that strong satisfiability implies projectivity. Lemma 11 shows that closure under the Visser rules (to be defined below) is a necessary and sufficient condition for strong satisfiability. Together with Theorem 1 it therefore proves Theorem 2.

The proof of Lemma 11 is fairly straightforward and will be discussed in Section 6 , but the proof of Theorem 1 needs some clarification, which we provide in the remainder of this section.

Instead of formulas it is convenient to work with a set of sequents $\mathcal{G}$ and corresponding formula $G=I(\mathcal{G})$. Now suppose $\sigma_{G}$ is a composition $\sigma_{m} \ldots \sigma_{1}$ of 
substitutions of the form $\sigma_{I}^{G}$ and we wish to prove $\vdash \sigma_{G} G$, that is, $\vdash \sigma_{G} S_{1}$ for all $S_{1} \in \mathcal{G} . \bar{\sigma}_{i}$ denotes $\sigma_{m} \ldots \sigma_{i}$. Thus $\bar{\sigma}_{1}=\sigma_{G}$.

As $G \vdash \sigma_{j} \leftrightarrow \iota$, for $i<j$ we have $\vdash \bar{\sigma}_{j} G \rightarrow \bar{\sigma}_{i} G$. In particular, $\vdash \bar{\sigma}_{2} G \rightarrow \bar{\sigma}_{1} G$, and as $S_{1} \in \mathcal{G}$, also $\vdash \bar{\sigma}_{2} G \rightarrow \bar{\sigma}_{1} S_{1}$. To show $\vdash \sigma_{G} S_{1}$, that is $\sigma_{G} S_{1}^{a} \vdash \sigma_{G} S_{1}^{c}$, it therefore suffices to show $\sigma_{G} S_{1}^{a} \vdash \bar{\sigma}_{2} G$. For the latter, we have to show that $\sigma_{G} S_{1}^{a} \vdash \bar{\sigma}_{2} S_{2}$ for all $S_{2} \in \mathcal{G}$. This would follow from $\sigma_{G} S_{1}^{a}, \bar{\sigma}_{2} S_{2}^{a} \vdash \bar{\sigma}_{3} G$. Continuing in this manner, we see that $\sigma_{G} S_{1}^{a}, \bar{\sigma}_{2} S_{2}^{a}, \ldots, \bar{\sigma}_{j} S_{j}^{a} \vdash \bar{\sigma}_{j+1} G$ is a sufficient condition for $\vdash \sigma_{G} G$, for every $j<m$.

As we will see, Proposition 1 , for any $I$ such that $\sigma_{j+1}$ is equal to $\sigma_{I}$, if $v_{I}\left(S^{a v}, S_{\Sigma_{S_{1}, \ldots, S_{j}}^{a i}}^{a} \Rightarrow S^{c} \cap \Sigma_{S_{1}, \ldots, S_{j}}\right)=1$, then $\sigma_{G} S_{1}^{a}, \ldots, \bar{\sigma}_{j} S_{j}^{a} \vdash \bar{\sigma}_{j+1} S$. To prove $\vdash \sigma_{G} G$ it therefore suffices to show that for every sequence $S_{1}, \ldots, S_{h}$ of sequents from $\mathcal{G}$, there is an extension $S_{1}, \ldots, S_{h}, \ldots, S_{j}$ of sequents in $\mathcal{G}$ such that $v_{I}\left(S^{a v}, S_{\Sigma_{S_{1}, \ldots, S_{j}}}^{a i} \Rightarrow S^{c} \cap \Sigma_{S_{1}, \ldots, S_{j}}\right)=1$. As we will see below, strong satisfiability implies exactly this.

\section{Substitutions and valuations}

The discussion above serves as a motivation for the notions introduced in this section. In this and the next section we consider an arbitrary finite set $\mathcal{G}$ of irreducible sequents, and corresponding formula $G=I(\mathcal{G})$, and assume the atoms that occur in $\mathcal{G}$ to be $\left\{p_{1}, \ldots, p_{n_{\mathcal{G}}}\right\}$. Most definitions are relative to $\mathcal{G}$ but for simplicity we do not always indicate this in our notation. $\mathcal{G}^{\star}$ consists of those sequents $S \in \mathcal{G}$ such that $\left(S^{a} \cap S^{c} \cap \mathcal{P}\right)$ is empty. Note that all sequents in $\mathcal{G}$ that are not in $\mathcal{G}^{\star}$ are derivable.

We fix an arbitrary enumeration $J_{1}, \ldots, J_{2^{n} \mathcal{G}}$ of all subsets of $\left\{p_{1}, \ldots, p_{n_{\mathcal{G}}}\right\}$, and let $I$ range over subsets of $\left\{p_{1}, \ldots, p_{n_{\mathcal{G}}}\right\}$. Given a set $I$ the valuation $v_{I}$ has been defined above, and $\sigma_{I}$ denotes $\sigma_{I}^{G}$, also defined at the beginning of Section 3 . We extend it to a valuation for sequents $S$ relative to a set of sequents $\mathcal{S}: S$ is strongly satisfiable with respect to $\mathcal{S}$ if

$$
\bar{v}_{I}(S \mid \mathcal{S}) \equiv_{d e f} v_{I}\left(S^{a v}, S_{\Sigma_{\mathcal{S}}}^{a i} \Rightarrow S^{c} \cap \Sigma_{\mathcal{S}}\right)=1 .
$$

If $S_{\Sigma_{\mathcal{S}}}^{a i}$ is empty, the right side is read as $v_{I}\left(S^{a v} \Rightarrow S^{c} \cap \Sigma_{\mathcal{S}}\right)=1$, similarly for $S^{a v}$ and $S^{c} \cap \Sigma_{\mathcal{S}}$. The valuations are extended to sets of sequents in the usual way: $\bar{v}_{I}\left(\mathcal{S}^{\prime} \mid \mathcal{S}\right)=1$ if and only if $\bar{v}_{I}(S \mid \mathcal{S})=1$ for all $S \in \mathcal{S}^{\prime}$. We write $\bar{v}_{I}(\mathcal{S})$ for $\bar{v}_{I}(\mathcal{S} \mid \mathcal{S})$. $\mathcal{G}$ is strongly satisfiable if for all $\mathcal{S} \subseteq \mathcal{G}^{\star}$ there is an $I$ such that $\bar{v}_{I}(\mathcal{S})=1$.

We use the following abbreviationa in this and the next section:

$$
g=2^{n_{\mathcal{G}}} \quad \sigma_{G} \equiv_{\text {def }}\left(\sigma_{J_{g}} \ldots \sigma_{J_{1}}\right)^{(|\mathcal{G}|+1)} .
$$

Thus $\sigma_{G}$ is the concatenation of $g(|\mathcal{G}|+1)$ substitutions. The $i$-th substitution in $\sigma_{G}$ (reading from right to left) is denoted by $\sigma_{i}$ and for $i<j, \sigma_{j} \ldots \sigma_{i}$ is denoted by $\sigma_{j, i}$. We denote $\sigma_{g(|\mathcal{G}|+1), i}=\sigma_{g(|\mathcal{G}|+1)} \ldots \sigma_{i}$ by $\bar{\sigma}_{i}$. For example, $\sigma_{2}=\sigma_{g+2}=\cdots=\sigma_{g|\mathcal{G}|+1}=\sigma_{J_{2}}, \bar{\sigma}_{1}=\sigma_{G}$, and $\bar{\sigma}_{g+1}=\bar{\sigma}^{|\mathcal{G}|}$. Note that for 
$i<j, \bar{\sigma}_{j}$ is the tail of $\bar{\sigma}_{i}$. We denote by $I_{i}$ the set $J_{j}$ such that $\sigma_{i}=\sigma_{J_{j}}$. For valuations we define: $v_{i} \equiv_{\text {def }} v_{I_{i}}$.

Lemma 1 For all $i<j: \vdash G \rightarrow\left(\iota \leftrightarrow \sigma_{i} \leftrightarrow \bar{\sigma}_{j, i}\right)$ and $\vdash \bar{\sigma}_{j} G \rightarrow \bar{\sigma}_{i} G$.

Proof The first equivalence in the first statement is clear. The second equivalence follows from this and the fact that $\vdash(B \leftrightarrow C) \rightarrow(A[B / p] \leftrightarrow A[C / p])$ for any atom $p$.

The first statement implies that $\vdash G \rightarrow \sigma_{j-1, i} G$, which implies $\vdash \bar{\sigma}_{j} G \rightarrow \bar{\sigma}_{i} G$.

Define

$$
F\left(i_{1}, \ldots, i_{j}, S_{1}, \ldots, S_{j}, A\right) \equiv_{d e f} \bar{\sigma}_{i_{1}} S_{1}^{a}, \bar{\sigma}_{i_{2}} S_{2}^{a}, \ldots \bar{\sigma}_{i_{j}} S_{j}^{a} \Rightarrow A .
$$

Proposition 1 For all $\mathcal{S}=\left\{S_{1}, \ldots, S_{j}\right\} \subseteq \mathcal{G}$ and all $1 \leq i_{1}, \ldots, i_{j} \leq g(|\mathcal{G}|+1)$, if $\bar{v}_{I}(\mathcal{S})=1$, then for all $S \in \mathcal{S}: \vdash F\left(i_{1}, \ldots, i_{j}, S_{1}, \ldots, S_{j}, \sigma_{I} S\right)$.

Proof First the case that $S^{a v} \backslash I$ or $S^{c} \cap \Sigma_{\mathcal{S}}$ is nonempty. In case $p \in S^{a v} \backslash I$, $\sigma_{I}(p)=G \wedge p$, and thus $\sigma_{I} S^{a}$ derives $G$, and therefore it dereives $S^{c}$ and $\sigma_{I} S^{c}$ by Lemma 1. Thus proving that $\sigma_{I} S$ is derivable, even without the assumptions. In case $p \in S^{c} \cap \Sigma_{\mathcal{S}}, \sigma_{I}(p)=G \rightarrow p$ and $\vdash G \wedge \wedge S_{h}^{a} \rightarrow p$. Hence also $F\left(i_{1}, \ldots, i_{j}, S_{1}, \ldots, S_{j}, \sigma_{I} p\right)$, which gives $\vdash F\left(i_{1}, \ldots, i_{j}, S_{1}, \ldots, S_{j}, \sigma_{I} S\right)$ as $p \in$ $S^{c}$.

Finally the remaining case: there is an implication $(p \rightarrow q) \in S^{a}$ with $p \in \Sigma_{\mathcal{S}}$, $p \in I$ and $q \notin I$. Thus $\bar{v}_{I}((p \rightarrow q) \mid \mathcal{S})=(G \rightarrow p) \rightarrow G \wedge q$. As $p \in \Sigma_{\mathcal{S}}$, $G \vdash \bigwedge_{h} S_{h}^{a} \rightarrow p$, and therefore Lemma 1 implies $\vdash \bigwedge_{h} \bar{\sigma}_{i_{h}} S_{h}^{a} \rightarrow(G \rightarrow p)$. Hence $\vdash \bigwedge_{h} \bar{\sigma}_{i_{h}} S_{h}^{a} \wedge \sigma_{I} S^{a} \rightarrow G \wedge q$. The fact that $S \in \mathcal{G}$ finally leads to $\vdash \bigwedge_{h} \bar{\sigma}_{i_{h}} S_{h}^{a} \wedge \sigma_{I} S^{a} \rightarrow \sigma_{I} S^{c}$, which is what we had to show.

\section{$5 \quad$ Unifiers}

In this section we show that every strongly satisfiable set of sequents $\mathcal{G}$ has $\sigma_{G}$ as a projective unifier. We need some terminology to be able to prove this theorem by backwards induction. A sequence of $m$ numbers followed by $m$ sequents $i_{1}, \ldots, i_{m}, S_{1}, \ldots, S_{m}$ is appropriate if $m \leq|\mathcal{G}|$,

$$
1=i_{1} \leq g<i_{2} \leq 2 g \leq \cdots<i_{m} \leq m g,
$$

and the sequents are distinct and belong to $\mathcal{G}$. It is $G$-sufficient if for all numbers $j$ such that $m g<j \leq(m+1) g$ and $\bar{v}_{j}\left(\left\{S_{1}, \ldots, S_{m}\right\}\right)=1$, the formula $F\left(i_{1}, \ldots, i_{m}, S_{1}, \ldots, S_{m}, \bar{\sigma}_{j} G\right)$ is derivable, where $F$ is defined in (1).

Lemma 2 If $\mathcal{G}$ is strongly satisfiable, then for every appropriate sequence $i_{1}, \ldots, i_{m}, S_{1}, \ldots, S_{m}$ and any number $k>0$ there exists a number $h$ such that $k g<h \leq(k+1) g$ and $\bar{v}_{h}\left(\left\{S_{1}, \ldots, S_{m}\right\}\right)=1$. 
Proof As $\mathcal{G}$ is strongly satisfiable, there is a $j \leq g$ such that $\bar{v}_{j}\left(\left\{S_{1}, \ldots, S_{m}\right\}\right)$ equals 1 . Since $v_{j}=v_{k g+j}$, the lemma follows.

Lemma 3 If $\mathcal{G}$ is strongly satisfiable then for all $m \leq|\mathcal{G}|$ : if all appropriate sequences of length $2 m$ are $G$-sufficient, then so are all appropriate sequences of length $2 m-2$.

Proof Consider an appropriate $i_{1}, \ldots, i_{m-1}, S_{1}, \ldots, S_{m-1}$ and let $j$ be such that $(m-1) g<j \leq m g$ and $\bar{v}_{j}\left(\left\{S_{1}, \ldots, S_{m-1}\right\}\right)=1$. We have to show that for all $S \in \mathcal{G}:$

$$
\vdash F\left(i_{1}, \ldots, i_{m-1}, S_{1}, \ldots, S_{m-1}, \bar{\sigma}_{j} S\right)
$$

If $S \in\left\{S_{1}, \ldots, S_{m-1}\right\}$, then (2) follows from Proposition 1. If, on the other hand, $S \notin\left\{S_{1}, \ldots, S_{m-1}\right\}$, then $i_{1}, \ldots, i_{m-1}, j, S_{1}, \ldots, S_{m-1}, S$ is an appropriate sequence of length $2 m$. By Lemma 2 there exists a number $h$ such that $m g<h \leq(m+1) g$ and $\bar{v}_{h}\left(\left\{S_{1}, \ldots, S_{m-1}, S\right\}\right)=1$. Therefore by $G$-sufficiency

$$
\vdash F\left(i_{1}, \ldots, i_{m-1}, j, S_{1}, \ldots, S_{m-1}, S, \bar{\sigma}_{h} G\right) .
$$

Since $\vdash \bar{\sigma}_{h} G \rightarrow \bar{\sigma}_{j} G$ and $S \in \mathcal{G}$, this implies that

$$
\vdash F\left(i_{1}, \ldots, i_{m-1}, j, S_{1}, \ldots, S_{m-1}, S, \bar{\sigma}_{j} S\right) .
$$

Hence $\vdash F\left(i_{1}, \ldots, i_{m-1}, S_{1}, \ldots, S_{m-1}, \bar{\sigma}_{j} S\right)$, which is what we had to show.

Lemma 4 If $S \in \mathcal{G}$ and $1, S$ is $G$-sufficient, then $\vdash \bar{\sigma} S$.

Proof By Lemma 2 there exists an $i \leq 2 g$ such that $\bar{v}_{i}(\{S\})=1$. Hence $\vdash \bar{\sigma}_{1} S^{a} \rightarrow \bar{\sigma}_{i} G$. Since $\vdash \bar{\sigma}_{i} G \rightarrow \bar{\sigma}_{1} G$ by Lemma 1 , this gives $\vdash \bar{\sigma}_{1} S^{a} \rightarrow \bar{\sigma}_{1} G$. As $S \in \mathcal{G}, \vdash \bar{\sigma}_{1} S$ follows, that is, $\vdash \sigma_{G} S$.

Lemma 5 Every appropriate sequence of length $2|\mathcal{G}|$ is $G$-sufficient.

Proof Let $|\mathcal{G}|=m$ and consider an appropriate sequence $i_{1}, \ldots, i_{m}, S_{1}, \ldots, S_{m}$ and let $j$ be such that $m g<j \leq(m+1) g$ and $\bar{v}_{j}\left(\left\{S_{1}, \ldots, S_{m}\right\}\right)=1$. Because $m=|\mathcal{G}|$ and the $S_{i}$ are distinct, $\left\{S_{1}, \ldots, S_{m}\right\}=\mathcal{G}$. Therefore Proposition 1 gives $\vdash F\left(i_{1}, \ldots, i_{m}, S_{1}, \ldots, S_{m}, \bar{\sigma}_{j} G\right)$.

Theorem 1 If $\mathcal{G}$ is strongly satisfiable, then $\vdash \sigma_{G} G$.

Proof By Lemma 5 every appropriate sequence of length $2|\mathcal{G}|$ is $G$-sufficient. By repeated application of Lemma 3 it follows that $1, S$ is $G$-sufficient for every $S \in \mathcal{G}$. This implies $\vdash \sigma_{G} S$ by Lemma 4 . 


\section{Rules and satisfiability}

In this section we show that closure under the Visser rules, (in sequent notation)

$$
\frac{\{\Gamma \rightarrow \Delta\}}{\left\{\Gamma \Rightarrow A \mid A \in \Gamma^{a} \cup \Delta\right\}} \bar{V} \quad(\Gamma \text { implications only })
$$

implies strong satisfiability, thus proving by Theorem 1 that closure under the Visser rules implies projectivity. We make use of a property, being closed, that is equivalent to being closed under the Visser rules, Lemma 10, but easier to apply. Before giving the formal definition, we treat two examples indicating how closure conditions can imply satisisfiability. In both examples $\mathcal{G}$ is a consistent set of sequents, which means that it does not derive the empty sequent.

Given $S \in \mathcal{G}$, the only way in which $S=(\Gamma \Rightarrow \Delta)$ cannot be strongly satisfiable with respect to $\mathcal{G}$, which means $\bar{v}_{I}(S \mid \mathcal{G})=v_{I}\left(S^{a v}, S_{\Sigma_{\mathcal{G}}}^{a i} \Rightarrow S^{v} \cap \Sigma_{\mathcal{G}}\right)=0$ for all $I$, is if $\Gamma$ consists of implications such that $\Gamma^{a} \cap \Sigma_{\mathcal{G}}=\Delta \cap \Sigma_{\mathcal{G}}=\emptyset$. However, if $\mathcal{G}$ is closed under the Visser rules, it contains $(\Gamma \Rightarrow p)$ for at least one $p \in \Gamma^{a} \cup \Delta$. But then $p \in \Sigma_{\mathcal{G}}$, contradicting $\Gamma^{a} \cap \Sigma_{\mathcal{G}}=\Delta \cap \Sigma_{\mathcal{G}}=\emptyset$. This shows that for single $S \in \mathcal{G}, \mathcal{G}$ being closed under the Visser rules implies that $S$ is strongly satisfiable with respect to $\mathcal{G}$.

If we consider more than one sequent, we need the notion of being closed, as illustrated by the following example. Consider sequents $S_{1}, S_{2} \in \mathcal{G}$ such that $S_{1}=(\Gamma, q \rightarrow r \Rightarrow q)$ and $S_{2}=(q \Rightarrow \Delta)$ and $\Gamma$ consists of implications not equal to $(s \rightarrow s)$ and $q \notin \Delta$. If $\left\{S_{1}, S_{2}\right\}$ is not strongly satisfiable with respect to $\mathcal{G}$, then $\Gamma^{a} \cup \Delta$ does not contain elements from $\Sigma_{\mathcal{G}}$ If $\mathcal{G}$ is closed under the Visser rules and as it derives $\Gamma, q \rightarrow r \Rightarrow \Delta$, it also derives $\Gamma \Rightarrow p$ for at least one $p \in \Gamma^{a} \cup\{q\} \cup \Delta$. This, however, does no lead to a contradiction as in the case above, as $q$ can be taken for $p$. But as we will show in Lemma 10, closure under the Visser rules implies being closed, which implies that $\mathcal{G}$ derives $\Gamma \Rightarrow p$ for at least one $p \in \Gamma^{a} \cup \Delta$, which is a contradiction. Thus showing that closure under the Visser rules of $\mathcal{G}$ implies that $\left\{S_{1}, S_{2}\right\}$ is strongly satisfiable with respect to $\mathcal{G}$.

The seemingly stronger notion of being closed is defined as follows. We use the following notation for sets of formulas in which some implications are replaced by their antecedents:

$$
\Gamma_{I} \equiv_{\text {def }} I \cup \Gamma \backslash\{(p \rightarrow q) \in \Gamma \mid p \in I\} .
$$

$\mathcal{G}$ is closed if for all irreducible sequents $(\Gamma \Rightarrow \Delta)$ in $\mathcal{L}_{\mathcal{G}}$ such that $\Gamma$ does not contain atoms, $\mathcal{G}$ is closed under the rule

$$
\left\{\Gamma_{J} \Rightarrow \Delta \mid J \subseteq I\right\} /\left\{\Gamma \Rightarrow p \mid p \in\left(\Gamma^{a} \backslash I\right) \cup \Delta\right\}
$$

Note that the Visser rules are a special instance of the above rule, namely for $I=\emptyset$. 


\subsection{Resolution}

To prove that being closed implies being strongly satisfiable, we argue by contradiction. Assuming that $\mathcal{G}$ is not strongly satisfiable, which means that $\bar{v}_{I}(\mathcal{G})=0$ for all $I \subseteq \Sigma_{\mathcal{G}}$, we use a resolution proof on the sequents $\left(S^{a v}, S_{\Sigma_{\mathcal{G}}}^{a i} \Rightarrow S^{v} \cap \Sigma_{\mathcal{G}}\right)$ for $S \in \mathcal{G}$, to conclude that $\mathcal{G}$ is not closed. The following lemmas about resolution proofs are needed to draw that conclusion.

Resolution proofs in the usual sense correspond to sequent derivations in which every sequent contains only atoms, and every inference is a cut. The only difference between resolution proofs and the $\Sigma$-resolution proofs we consider below is that in our case we use irreducible sequents. And although the only inference rules are cuts on atoms, the implications are present as additional information that will be used in the next lemmas.

A sequent $S$ is full in $\Sigma$ if $S^{a a} \cap \Sigma \subseteq S^{c} . \mathcal{F}_{\mathcal{G}}^{\Sigma}$ is the set of sequents that are full in $\Sigma$ and that can be obtained from $S$ by left implications in $\Sigma$ :

$$
\mathcal{F}_{S}^{\Sigma} \equiv_{\text {def }}\left\{S^{a} \backslash \Pi, \Pi^{c} \Rightarrow S^{c},\left(S^{a} \backslash \Pi\right)^{a} \cap \Sigma \mid \Pi \subseteq S_{\Sigma}^{a i}\right\} \quad \mathcal{F}_{\mathcal{G}}^{\Sigma} \equiv_{\text {def }} \cup\left\{\mathcal{F}_{S}^{\Sigma} \mid S \in \mathcal{G}\right\} .
$$

Lemma $6 F_{\mathcal{G}}^{\Sigma} \dashv-G$. For every $I \subseteq \Sigma: v_{I}\left(\mathcal{F}_{S}^{\Sigma}\right)=1$ implies $v_{I}(S)=1$.

Given a set of atoms $\Sigma$, an $\Sigma$-resolution proof of $S$ from $\mathcal{G}$ is a finite binary tree labelled with sequents: the leafs are (labelled with) sequents in $\mathcal{F}_{\mathcal{G}}^{\Sigma}$, the root is $S$, and a sequent at an inner node is the result of a cut in $\Sigma$ on the two sequents immediately above it. $\mathcal{C}_{\mathcal{R}}$ is the set of cut formulas that occur in $\mathcal{R}$. Thus $\mathcal{C}_{\mathcal{R}} \subseteq \Sigma$.

Lemma 7 If $v_{I}(\mathcal{G})=0$ for all $I \subseteq \Sigma$, then there exists a $\Sigma$-resolution proof from $\mathcal{G}$ of a sequent $S$ such that $S^{\overline{a v}} \cup\left(S^{c} \cap \Sigma\right)=\emptyset$.

Proof Let $\bar{\Sigma}$ be the complement of $\Sigma$ in $\mathcal{P}$. Consider

$$
\mathcal{H}=\left\{\left(S^{a v}, S^{a i} \backslash S_{\bar{\Sigma}}^{a i} \Rightarrow S^{c} \cap \Sigma\right) \mid S \in \mathcal{G}\right\} .
$$

Suppose $v_{I}(\mathcal{G})=0$ for all $I \subseteq \Sigma$. This implies that $\mathcal{H}$ is classically inconsistent, as all positive atoms in the sequents in $\mathcal{H}$ belong to $\Sigma$. From Lemma 6 it follows that $\mathcal{F}_{\mathcal{H}}^{\mathcal{P}}$ is classically inconsistent. By the completeness of resolution refutations for classical logic, there exists a $\mathcal{P}$-resolution proof from $\mathcal{F}_{\mathcal{H}}^{\mathcal{P}}$ of a sequent that does not contain atoms. Since all cut formulas belong to $\Sigma$, this proof corresponds to an $\Sigma$-resolution proof from $\mathcal{G}$ of a sequent $S$ for which $S^{a v} \cup\left(S^{c} \cap \Sigma\right)=\emptyset$.

Lemma 8 If $\mathcal{R}$ is a $\Sigma$-resolution proof of $S$, then

$$
\left(S^{a a} \cap \Sigma\right) \backslash \mathcal{C}_{\mathcal{R}} \subseteq S^{c} .
$$

Proof With induction to the number of cuts in $\mathcal{R}$. If $\mathcal{R}$ does not contain cuts, then $S \in \mathcal{F}_{\mathcal{G}}^{\Sigma}$, which clearly implies the statement. Suppose that $\mathcal{R}$ contains 
cuts, that $S=(\Gamma, \Pi \Rightarrow \Delta, \Lambda)$, and that the lowest cut is:

$$
\frac{\Gamma \Rightarrow p, \Delta \quad p, \Pi \Rightarrow \Lambda}{\Gamma, \Pi \Rightarrow \Delta, \Lambda}
$$

Consider $q \in\left(S^{a a} \cap \Sigma\right) \backslash \mathcal{C}_{\mathcal{R}}$. Since $p \in \mathcal{C}_{\mathcal{R}}, q \neq p$. Note that $q \in \Gamma^{a}$ or $q \in \Pi^{a}$. In both cases the induction hypothesis implies that $q \in \Delta \cup \Lambda$, which is what we had to show.

Lemma 9 For all $\Sigma$-resolution proofs $\mathcal{R}$ of $S \in \mathcal{G}$, and all $I \subseteq \mathcal{C}_{\mathcal{R}}$ :

$$
G \vdash\left(S_{I}^{a} \Rightarrow S^{c}\right) \text { or } I \cap S^{c} \neq \emptyset \text {. }
$$

Proof With induction to the number of cuts in $\mathcal{R}$. If there are no cuts, the lemma follows immediately. Suppose there are cuts in $\mathcal{R}$ and consider the lowest cut

$$
\frac{\Gamma \Rightarrow p, \Delta \quad p, \Pi \Rightarrow \Lambda}{\Gamma, \Pi \Rightarrow \Delta, \Lambda}
$$

Let $S$ be the conclusion, and let $\mathcal{R}^{\prime}$ and $\mathcal{R}^{\prime \prime}$ be resolution proofs of respectively the left and the right premise. Consider $I \subseteq \mathcal{C}_{\mathcal{R}}$ and let $J=I \cap \Gamma^{a}$ and $H=I \cap \Pi^{a}$ and assume that $I \cap(\Delta \cup \Lambda)$ is empty, otherwise we are done immediately. If there exists a $q \in H \backslash \mathcal{C}_{\mathcal{R}^{\prime \prime}}$, then $q \in \Lambda$ by Lemma 8 , and thus $S^{c} \cap I$ is not empty, which contradicts our assumptions. Therefore $H \subseteq \mathcal{C}_{\mathcal{R}^{\prime \prime}}$. Thus $G$ derives $\left(p, \Pi_{H} \Rightarrow \Lambda\right)$ by the induction hypothesis.

If $p \in I$, this implies that $\Pi_{H} \cup\{p\} \subseteq \Pi_{I}$, and thus $G$ derives $\left(\Gamma_{I} \Pi_{I} \Rightarrow \Delta, \Lambda\right)$. If, on the other hand, $p \notin I$, then $p \notin J$. Thus $J \subseteq \mathcal{C}_{\mathcal{R}^{\prime}}$. Hence $G$ derives $\left(\Gamma_{J} \Rightarrow p, \Delta\right)$ by the induction hypothesis. Thus it derives $\left(\Gamma_{J}, \Pi_{H} \Rightarrow \Delta, \Lambda\right)$ and whence $\left(\Gamma_{I}, \Pi_{I} \Rightarrow \Delta, \Lambda\right)$.

Lemma $10 \mathcal{G}$ is closed if and only if $\mathcal{G}$ is closed under the multi-conclusion Visser rules.

Proof The direction from left to right follows from the observation at the beginning of this section. For the other direction, we first treat the case that disjunction is in the language. Consider $I$ and $(\Gamma \Rightarrow \Delta)$. Let $\Gamma^{\langle H, \Delta\rangle}$ be the result of replacing every $p \in \Gamma^{a} \cap H$ by $(p \wedge \bigvee \Delta)$

First we show that with induction to $|H|$ that for all $J, H \subseteq I$ :

$$
\left\{\left(\Gamma_{J} \Rightarrow \Delta\right) \mid J \subseteq I\right\} \vdash_{\mathrm{L}} \Gamma_{J}^{\langle H, \Delta\rangle} \leftrightarrow \Gamma_{J}
$$

Since the $\leftarrow$ part is trivial, it remains to show

$$
\left\{\left(\Gamma_{J} \Rightarrow \Delta\right) \mid J \subseteq I\right\} \vdash_{\mathrm{L}} \Gamma_{J}^{\langle H, \Delta\rangle} \Rightarrow \Gamma_{J} .
$$

If $H=\emptyset$, then $\Gamma_{J}^{\langle H, \Delta\rangle}=\Gamma_{J}$. Suppose $|H|>0$, and consider $p_{i} \in H$, such that $\left(p_{i} \rightarrow q\right) \in \Gamma_{J}$ (if there is more than one implication in $\Gamma$ with antecedent $p_{i}$, the argument remains the same). By the induction hypothesis

$$
\left\{\left(\Gamma_{J} \Rightarrow \Delta\right) \mid J \subseteq I\right\} \vdash_{\mathrm{L}} \Gamma_{J \cup\{i\}}^{\langle H \backslash\{i\}, \Delta\rangle} \Rightarrow \Gamma_{J \cup\{i\}} .
$$


Since $\left\{\left(\Gamma_{J} \Rightarrow \Delta\right) \mid J \subseteq I\right\} \vdash_{\llcorner} \Gamma_{J \cup\{i\}} \Rightarrow \Delta$, this implies that

$$
\left\{\left(\Gamma_{J} \Rightarrow \Delta\right) \mid J \subseteq I\right\} \vdash_{\llcorner} \Gamma_{J \cup\{i\}}^{\langle H \backslash\{i\}, \Delta\rangle} \Rightarrow \Delta .
$$

As $\left(p_{i} \wedge \bigvee \Delta \rightarrow q\right) \in \Gamma_{J}^{\langle H, \Delta\rangle},\left(p_{i} \rightarrow q\right) \notin \Gamma_{J \cup\{i\}}$ and $p_{i} \in \Gamma_{J \cup\{i\}}$, this gives

$$
\left\{\left(\Gamma_{J} \Rightarrow \Delta\right) \mid J \subseteq I\right\} \vdash_{\mathrm{L}} \Gamma_{J}^{\langle H, \Delta\rangle} \Rightarrow\left(p_{i} \rightarrow q\right) .
$$

Thus $\left\{\left(\Gamma_{J} \Rightarrow \Delta\right) \mid J \subseteq I\right\} \vdash_{\mathrm{L}} \Gamma_{J}^{\langle H, \Delta\rangle} \Rightarrow \Gamma_{J}^{\langle H \backslash\{i\}, \Delta\rangle}$, and the induction hypothesis gives (3).

Thus we have shown (3), and in particular

$$
\left\{\left(\Gamma_{J} \Rightarrow \Delta\right) \mid J \subseteq I\right\} \vdash_{\mathrm{L}} \Gamma^{\langle I, \Delta\rangle} \leftrightarrow \Gamma .
$$

The following observations now prove the lemma:

$$
\begin{aligned}
& \left.\left(\Gamma_{J} \Rightarrow \Delta\right) \mid J \subseteq I\right\} \quad \vdash \quad \Gamma \Rightarrow \Delta \\
& \vdash \quad \Gamma^{\langle I, \Delta\rangle} \Rightarrow \Delta \\
& \sim \quad\left\{\Gamma^{\langle I, \Delta\rangle} \Rightarrow p \mid p \in\left(\Gamma^{\langle I, \Delta\rangle}\right)^{a} \cup \Delta\right\} \\
& \vdash \quad\left\{\Gamma \Rightarrow p \mid p \in\left(\Gamma^{\langle I, \Delta\rangle}\right)^{a} \cup \Delta\right\} \\
& \vdash \quad\left\{\Gamma \Rightarrow p \mid p \in \Gamma^{a} \backslash I \cup \Delta\right\}
\end{aligned}
$$

The proof for the case that disjunction is not present is similar: we have to replace $(p \wedge \bigvee \Delta \rightarrow q)$ by $\bigwedge_{r \in \Delta}(p \wedge r \rightarrow q)$.

Lemma 11 If $\mathcal{G}$ is closed under $\bar{\nabla}$, it is strongly satisfiable.

Proof Suppose that $\mathcal{G}$ is not strongly satisfiable. Observe that this implies in particular that for all $I \subseteq \Sigma_{\mathcal{G}}, v_{I}(\mathcal{G})=0$. By Lemma 7 there is a $\Sigma_{\mathcal{G}}$-resolution proof $\mathcal{R}$ of a sequent $S \in \mathcal{G}$ for which $S^{a v} \cup\left(S^{c} \cap \Sigma_{\mathcal{G}}\right)=\emptyset$. Thus $S^{a}$ consists of implications. Since $\mathcal{C}_{\mathcal{R}} \subseteq \Sigma_{\mathcal{G}}$, Lemma 9 implies that $G$ derives $S_{I}^{a} \Rightarrow S^{c}$ for all $I \subseteq \mathcal{C}_{\mathcal{R}}$. If $\mathcal{G}$ would be closed, it would derive $\left(S^{a} \Rightarrow p\right)$ for some $p \in S^{a a} \backslash \mathcal{C}_{\mathcal{R}} \cup S^{c}$. Hence $p \in \Sigma_{\mathcal{G}}$. Thus $p \in S^{c}$ by Lemma 8, which contradicts that $S^{c} \cap \Sigma_{\mathcal{G}}$ is empty. Therefore $\mathcal{G}$ is not closed, and thus not closed under $\overline{\mathrm{V}}$ by Lemma 10 .

Theorem 1 and Lemma 11 immediately give the following.

Theorem 2 If $\mathcal{G}$ is closed under $\overline{\mathrm{V}}$, then $G$ is projective with projective unifier $\sigma_{G}$.

IPC is the only intermediate logic with the disjunction property for which all multi-conclusion Visser rules are admissible [16]. In this logic the closure condition is a sufficient and necessary condition for strongly satisfiability.

Proposition 2 In IPC, $\mathcal{G}$ is closed under $\overline{\mathrm{V}}$ if and only if $\mathcal{G}$ is strongly satisfiable. 
Proof The direction from left to right is Lemma 11. The other direction uses that $\overline{\mathrm{V}}$ is admissible in IPC. Suppose that $\mathcal{G}$ is $\Sigma_{\mathcal{G}}$-satisfiable. Hence $\sigma_{G}$ is a projective unifier of $G$ by Theorem 1. Let $S / S^{\prime}$ be an instance of $\overline{\mathrm{V}}$ such that $G$ derives $S$. Thus $\sigma_{G} S$ is derivable in IPC, and by the admissibility of $\overline{\mathrm{V}}$ in IPC, so is $\sigma_{G} S^{\prime}$. Therefore $G$ derives $S^{\prime}$.

\section{$7 \quad$ Projective approximations}

In the following we will show that every sequent $S$ has a projective approximation. First we show that it can can be approximated by a set of irreducible sequents $\mathcal{G}_{S}$, in the sense that $S \Vdash G_{S} \Vdash G$, where $\Vdash$ is broad derivability, an extension of derivability defined as $S \nVdash S^{\prime}$ if and only if there is a substitution $\sigma$ that is the identity on $\mathcal{L}_{S}$ such that $S \vdash \sigma S^{\prime}$. We need this notion because in the construction of the approximation fresh atoms may be introduced. The corresponding extension of admissibility is broad admissibility defined as: $\mathcal{G} H \mathcal{H}$ if and only if every unifier of all sequents in $\mathcal{G}$ can be extended to a unifier of at least one sequent in $\mathcal{H}$.

We will use the language of rewrite systems [1] by characterizing irreducible sequents as normal forms of the rewrite system given below. Intuitively, this system deconstructs the formula along its syntactical structure, in most cases according to the rules of a standard Gentzen calculus, maintaining provability from right-to-left and broad derivability in the other direction. The rewrite relation will be well-founded (or terminating), hence we can easily obtain a finite set of irreducible sequents "approximating" another set of sequents in a sense to be made precise below. Dzik and Wojtylak use a similar method in [7]. Recall that $\mathcal{S}$ and $\mathcal{T}$ stand for finite sets of sequents. In the definition below we write $\mathcal{S}[\mathcal{T}]$ to mean $\mathcal{S} \cup \mathcal{T}$ where $\mathcal{S}$ and $\mathcal{T}$ do not overlap. The elements of $\mathcal{S}$ and $\mathcal{T}$ are divided by semi-columns rather than commas, so as not to confuse them with the commas that occur in the sequents.

Syntactic deconstruction is defined as the following rewrite system on sets of sequents. In the rules $\rightarrow \Rightarrow$ and $\Rightarrow \rightarrow$ the variables $p$ and $q$ are understood to be fresh in the usual sense, and in the rule $\rightarrow \Rightarrow$ it is assumed that $A \rightarrow B$ is not atomic.

$$
\begin{array}{rrll}
\perp \Rightarrow & \mathcal{S}[\perp, \Gamma \Rightarrow \Delta] & \longmapsto \mathcal{S} \\
\Rightarrow \perp & \mathcal{S}[\Gamma \Rightarrow \perp, \Delta] & \longmapsto \mathcal{S}[\Gamma \Rightarrow \Delta] \\
\wedge \Rightarrow & \mathcal{S}[A \wedge B, \Gamma \Rightarrow \Delta] & \longmapsto \mathcal{S}[A, B, \Gamma \Rightarrow \Delta] \\
\Rightarrow \wedge & \mathcal{S}[\Gamma \Rightarrow \Delta, A \wedge B] & \longmapsto \mathcal{S}[\Gamma \Rightarrow \Delta, A ; \Gamma \Rightarrow \Delta, B] \\
\vee \Rightarrow & \mathcal{S}[A \vee B, \Gamma \Rightarrow \Delta] & \longmapsto \mathcal{S}[A, \Gamma \Rightarrow \Delta ; B, \Gamma \Rightarrow \Delta] \\
\Rightarrow \vee & \mathcal{S}[\Gamma \Rightarrow \Delta, A \vee B] & \longmapsto \mathcal{S}[\Gamma \Rightarrow \Delta, A, B] \\
\rightarrow \Rightarrow & \mathcal{S}[A \rightarrow B, \Gamma \Rightarrow \Delta] & \longmapsto \mathcal{S}[p \Rightarrow A ; p \rightarrow q, \Gamma \Rightarrow \Delta ; B \Rightarrow q] \\
\Rightarrow & \mathcal{S}[\Gamma \Rightarrow \Delta, A \rightarrow B] & \longmapsto \mathcal{S}[\Gamma \Rightarrow \Delta, p ; p, A \Rightarrow B]
\end{array}
$$

The fresh variables $p$ and $q$ are such that they have an empty intersection with the sets $\operatorname{var}(\mathcal{S}), \operatorname{var}(\Gamma \Rightarrow \Delta), \operatorname{var}(A)$ and $\operatorname{var}(B)$. We can extend the set 
of variables which $p$ and $q$ are not supposed to hit by any finite amount of variables, which we will need to do later on.

Proposition 3 If $\mathcal{S} \longmapsto \mathcal{T}$, then $\bigwedge \mathcal{S} \Vdash \wedge \mathcal{T} \vdash \wedge \mathcal{S}$.

Proof In case $\longmapsto$ is one of the first six possibilities, the statement is clear, as in this case even $\bigwedge \mathcal{S} \vdash \wedge \mathcal{T}$. In case it is $\rightarrow \Rightarrow$, consider $\mathcal{S}[A \rightarrow B, \Gamma \Rightarrow \Delta]$. Then the substituion $\sigma$ that maps $p$ to $A$ and $q$ to $B$ is such that $\mathcal{S}[A \rightarrow B, \Gamma \Rightarrow \Delta]$ derives $\sigma \mathcal{S}[\sigma(p \Rightarrow A) ; \sigma(p \rightarrow q), \sigma \Gamma \Rightarrow \sigma \Delta ; \sigma(B \Rightarrow q)]$. The case $\Rightarrow \rightarrow$ is similar.

Corollary 1 For every set of sequents $\mathcal{S}$ there exists a set of irreducible sequents $\mathcal{G}$ such that $\bigwedge \mathcal{S} \Vdash \wedge \mathcal{G} \vdash \wedge \mathcal{S}$.

To show that every sequent $S$ has a projective approximation, we show that every finite set $\mathcal{G}$ of irreducible sequents has a projective approximation consisting of $\left\{\mathcal{G}_{1}, \ldots, \mathcal{G}_{m}\right\}$, where all $\mathcal{G}_{i}$ are finite sets of irreducible sequents in $\mathcal{L}_{\mathcal{G}}$ closed under the Visser rules. Theorem 2 implies that the $G_{i}$ are indeed projective.

We need the following relation $\stackrel{n}{\sim}$ on sets of irreducible sequents. For every set of irreducible sequents $\mathcal{G}$ and any irreducible sequent $\Gamma \Rightarrow \Delta$ in $\mathcal{L}_{\mathcal{G}}$ with $\Gamma$ implication-only of size at most $n$ and $\Delta$ of size at least two, such that $\mathcal{G} \vdash(\Gamma \Rightarrow \Delta)$ and for every $p \in \Gamma^{a} \cup \Delta$ we set

$$
\mathcal{G} \stackrel{n}{\rightarrow}(\mathcal{G} \cup\{\Gamma \Rightarrow \Delta, \Gamma \Rightarrow p\}) .
$$

Slightly ambiguous, we also use $\stackrel{n}{\sim}$ for the transitive closure of this relation. A set of sequents $\mathcal{G}$ is in $\stackrel{n}{\sim}$-normal form if there is no $\mathcal{H} \supset \mathcal{G}$ such that $\mathcal{G} \stackrel{n}{\sim} \mathcal{H}$. Note that $\stackrel{n}{\sim}$ is neither deterministic nor confluent, but it terminates in the following sense.

Lemma 12 For every set of irreducible sequents $\mathcal{G}$ and every $n \geq n_{\mathcal{G}}^{2}$ there are $\mathcal{G}_{1}, \ldots, \mathcal{G}_{m}$ in $\mathcal{L}_{\mathcal{G}}$ in $\stackrel{n}{\rightarrow}$-normal form such that $\mathcal{G} \stackrel{n}{\sim} \mathcal{G}_{i}$ and the $G_{i}$ are projective. If $\overline{\mathrm{V}}_{\mathrm{n}}$ is admissible, then $G \sim\left\{G_{1}, \ldots, G_{m}\right\}$ and $\bigvee G_{i} \vdash G$.

Proof Suppose $n \geq n_{\mathcal{G}}^{2}$. As $\mathcal{G}$ does not contain more than $n_{\mathcal{G}}$ atoms, the number of implications in an irreducible sequent are at most $n_{\mathcal{G}}^{2}$. Thus every possible $\stackrel{x}{\sim}$ step can be covered by $\stackrel{n}{\sim}$. Furthermore, there are at most finitely many irreducible sequents in $\mathcal{L}_{\mathcal{G}}$. Thus we can apply $\stackrel{n}{\sim}$ as long as possible and end up with sets of sequents in $\stackrel{n}{\sim}$-normal form that are closed under $\overline{\mathrm{V}}$. Theorem 2 implies that the $G_{i}$ are projective.

From the construction of the $\mathcal{G}_{i}$ it s clear that $\bigvee G_{i} \vdash G$ and that moreover $G \sim\left\{G_{1}, \ldots, G_{m}\right\}$ if $\bar{\nabla}_{\mathrm{n}}$ is admissible.

Theorem 3 For every sequent $S$, if $\overline{\mathrm{V}}_{i(S)}$ is admissible, there are sets of irreducible sequents $\mathcal{G}_{1}, \ldots, \mathcal{G}_{m}$ in $\mathcal{L}_{i(S)}$ such that $S \Vdash\left\{G_{1}, \ldots, G_{m}\right\}, \bigvee G_{i} \vdash S$ and the $G_{i}$ are projective. 
Proof Let $n$ be the number of atoms in $S$ and $m$ the number of implications in $S$. This implies that the number of atoms in the $\mathcal{G}$ that is obtained via Corollary 1 is at most $(n+2 m)^{2}$. Recall that $i(S)=(n+2 m)^{2}$. Thus the admissibility of $\overline{\mathrm{V}}_{i(S)}$ and Lemma 12 imply that there are sets of irreducible sequents $\mathcal{G}_{1}, \ldots, \mathcal{G}_{m}$ in $\mathcal{L}_{\mathcal{G}}$ such that $G \sim\left\{G_{1}, \ldots, G_{m}\right\}, \bigvee G_{i} \vdash G$ and the $G_{i}$ are projective. As $S \Vdash G \vdash S$, this gives $\bigvee G_{i} \vdash S$. And also $S \Vdash\left\{G_{1}, \ldots, G_{m}\right\}$, because every unifier of $S$ can be extended to a unifier of $G$, which is a unifier of some $G_{i}$ too.

Given a formula $A$, the set $\left\{G_{1}, \ldots, G_{m}\right\}$ in Theorem 3 for $S$ being $(\Rightarrow A)$ is the irreducible projective approximation of $A$.

Corollary 2 For every formula $A$, if $\overline{\mathrm{V}}_{i(A)}$ is admissible, then $A$ has an irreducible projective approximation.

\section{Unification types}

In this section we apply the results of the previous section to obtain results on unification and bases of admissible rules. The multi-conclusion Visser rules we have already encountered, and the Visser rules are the single-conclusion version of these rules in which the disjunction is internal (in sequent notation):

$$
\frac{\bigvee \Pi \vee(\bigwedge \Gamma \rightarrow \bigvee \Delta)}{\bigvee \Pi \vee \bigvee_{A \in \Gamma^{a} \cup \Delta}(\bigwedge \Gamma \rightarrow A)} \bigvee_{\mathrm{n}} \quad(\Gamma \text { implications only })
$$

$\mathrm{V}$ stands for the collection of all these rules, which play an important role in several intermediate logics that do not have the disjunction property.

The following theorem has been proved for the case L = IPC in [10,30], and for intermediate logics in [19]. In the latter paper the result for fragments is implicit.

Theorem 4 If $\mathrm{V}_{\mathrm{n}}$ is admissible in $\mathrm{L}$, then every consistent $A$ with $i(A) \leq n$ has a finite complete set of unifiers. If $\mathrm{L}$ does not contain disjunction then $A$ is projective.

Proof Given a consistent formula $A$, consider an irreducible projective approximation $\left\{G_{1}, \ldots, G_{m}\right\}$, which exists by Corollary 2 . Let $\tau_{i}$ be the projective unifier of $G_{i}$ and let $\sigma_{i}$ be its restriction to $\operatorname{var}(A)$. We show that $\left\{\sigma_{1}, \ldots, \sigma_{m}\right\}$ is a complete set of unifiers for $A$.

Consider a unifier $\sigma$ of $A$. As $A \Vdash\left\{G_{1}, \ldots, G_{m}\right\}, \sigma$ can be extended to a unifier $\tau$ of one of the $G_{i}$. Thus $\tau \leqslant \tau_{i}$. Observe that this implies that $\sigma \leqslant \sigma_{i}$, which is what we had to show.

If $\mathrm{L}$ does not contain disjunction, then $\stackrel{n}{\sim}$ cannot be applied, which means that $\mathcal{G}$, the set of irreducible sequents obtained via Corollary 1 such that $A \Vdash G \vdash A$, is already projective, say with projective unifier $\sigma_{G}$. Hence $\vdash \sigma_{G} A$. Thus $\vdash \sigma A$ for $\sigma$ the restriction of $\sigma_{G}$ to $\operatorname{var}(A)$. 
Using that for formulas of size at most $n, i(A) \leq 9 n^{2}$, we get the following corollary.

Corollary 3 If $\bar{V}_{9 n^{2}}$ is admissible, then the $n$-unification type of the logic is finitary.

IPC is the only intermediate logic with the disjunction property for which all multi-conclusion Visser rules are admissible [16]. Given this fact, the corollaries above immediately imply what has been proved by Mints, Ghilardi, and Rozière before.

Corollary 4 [10, 24, 30] Any fragment of IPC that contains conjunction and implication has finitary unification. If it does not contain disjunction it has unitary unification.

The fact that $\bar{V}_{\mathrm{n}+1}$ is admissible in the $n$-th Gabbay-De Jongh logic $\mathbf{T}_{\mathrm{n}}[9,16]$ implies the following.

Corollary 5 [14] Any fragment of $T_{n}$ that contains conjunction and implication has finitary $\sqrt{n+1 / 9}$-unification. If it does not contain disjunction it has unitary $\sqrt{n+1 / 9}$-unification under the same condition.

As another immediate corollary from Theorem 4 we obtain the following result by Mints (for IPC) and Minari and Wroński (for all intermediate logics).

Corollary $6[23,24]$ The implication-conjunction(-negation) fragment of any intermediate logic has unitary unification.

Using the fact that in Gödel-Dummett logic LC a disjunction $A \vee B$ is equivalent to $((A \rightarrow B) \rightarrow B) \wedge((B \rightarrow A) \rightarrow A)$ we can extend one direction of the result by Minari and Wroński [23] that an intermediate logic has projective unification (every formula has a pu) if and only if it contains LC, to fragments.

Corollary 7 Any fragment of LC that contains conjunction and implication has projective unification.

\section{References}

[1] F. Baader and T. Nipkow, Term Rewriting and All That, Cambridge University Press, 1998.

[2] F. Baader and W. Snyder, Unification theory, Handbook of automated reasoning, Elsevier, 2001.

[3] P. Cintula and G. Metcalfe, Structural completeness in fuzzy logics, Notre Dame Journal of Formal Logic 50(2), 2009, p.153-183.

[4] P. Cintula and G. Metcalfe, Admissible Rules in the Implication-Negation Fragment of Intuitionistic Logic, Submitted. 
[5] A.I. Citkin, On structurally complete superintuitionistic logics, Soviet Mathematics Doklady 19, 1978, p.816-819.

[6] W. Dzik, Splittings of Lattices of Theories and Unification Types, Proceedings of the Workshop on General Algebra 70, Verlag Johannes Heyn, 2006, p.71-81.

[7] W. Dzik and P. Wojtylak, Projective Unification in Modal Logic, Logic Journal of IGPL 20(1), 2011, p.121-153.

[8] W. Dzik and A. Wronński, Structural completeness of Gödel and Dummett's propositional calculi, Studia Logica 32, 1973, p.69-73.

[9] D. Gabbay and D.H.J. de Jongh, A Sequence of Decidable Finitely Axiomatizable Intermediate Logics with the Disjunction Property, Journal of Symbolic Logic 39(1), 1974, p.67-78.

[10] S. Ghilardi, Unification in intuitionistic logic, Journal of Symbolic Logic 64(2), 1999, p.859-880.

[11] S. Ghilardi, Best solving modal equations, Annals of Pure and Applied Logic 102, 2000, p.183-198.

[12] S. Ghilardi, A resolution/tableaux algorithm for projective approximations in IPC, Logic Journal of the IGPL 10(3), 2002, p.227-241.

[13] S. Ghilardi, Unification, Finite Duality and Projectivity in Varieties of Heyting Algebras, Annals of Pure and Applied Logic 127(1-3), 2004, p.99115.

[14] J. Goudsmit and R. Iemhoff, On unification and admissible rules in Gabbay-de Jongh logics, Annals of Pure and Applied Logic, to appear.

[15] R. Iemhoff, On the admissible rules of intuitionistic propositional logic, Journal of Symbolic Logic 66(1), 2001, p.281-294.

[16] R. Iemhoff, A(nother) characterization of intuitionistic propositional logic, Annals of Pure and Applied Logic 113 (1-3), 2001, p.161-173.

[17] R. Iemhoff, Intermediate logics and Visser's rules., Notre Dame Journal of Formal Logic 46 (1), 2005 p.65-81.

[18] R. Iemhoff, On Rules, Journal of Philosophical Logic, to appear.

[19] R. Iemhoff and G. Metcalfe, Proof theory for admissible rules, Annals of Pure and Applied Logic 159(1-2), 2009, p.171-186.

[20] R. Iemhoff and G. Metcalfe, Hypersequent systems for the admissible rules of modal and intermediate logics, Lecture Notes in Computer Science 5407 Proceedings of LFCS '09, S. Artemov and A. Nerode (eds.), Springer, 2009, p.230-245.

[21] E. Jeřábek, Admissible rules of modal logics, Journal of Logic and Computation 15(4), 2005, p.411-431.

[22] E. Jeřábek, Complexity of admissible rules, Archive for Mathematical Logic 46, 2007, p.73-92.

[23] P. Minari and A. Wroński, The property (HD) in intermediate logics: a partial solution of a problem of H. Ono, Reports on Mathematical Logic 22, 1988, p.21-25.

[24] G. Mints, Derivability of admissible rules, in Studies in constructive mathematics and mathematical logic, Zap. Nauchn. Sem. LOMI, V(32), Nauka, Leningrad, 1972, p.85-89.

[25] J.S. Olson, J.G. Raftery, and C.J.V. Alten, Structural completeness in substructural logics, Logic Journal of the IGPL 16(5), 2008, p.453-495.

[26] W.A. Pogorzelski, Structural completeness of the propositional calculus, Bull. Acad. Polon. Sci. Ser. Sci. Math. 19(5), 1971, p.349-351.

[27] T. Prucnal, On the structural completeness of some pure implicational propositional calculi, Studia Logica 32(1), 1973, p.45-50. 
[28] T. Prucnal, Structural completeness of Medvedev's propositional calculus, Reports on Mathematical Logic 6, 1976, p.103-105.

[29] T. Prucnal, On two problems of Harvey Friedman, Studia Logica 38(3), 1979, p.247-262.

[30] P. Rozière, Regles admissibles en calcul propositionnel intuitionniste, $\mathrm{PhD}$ thesis, Université Paris VII, 1992.

[31] V. Rybakov, Admissibility of Logical Inference Rules, Elsevier, 1997.

[32] V. Rybakov, Writing out Best Unifiers in Intuitionistic Logic for Formulas with Coefficients, Logic Journal of the IGPL, 2012, to appear.

[33] F. Wolter and M. Zakharyaschev, Undecidability of the Unification and Admissibility Problems for Modal and Description Logics, ACM Transactions on Computational Logic 9(4), article 25, 2008.

[34] A. Wroński, Transparent Unification Problem, Reports on Mathematical Logic 29, 1995, p.105107. 